\title{
NODAL SOLUTIONS FOR NONLINEAR NONHOMOGENEOUS NEUMANN EQUATIONS
}

\author{
Sergiu Aizicovici - Nikolaos S. Papageorgiou — Vasile Staicu
}

\begin{abstract}
We consider a nonlinear Neumann problem driven by a nonhomogeneous differential operator with a Caratheodory reaction which is $(p-1)$-sublinear near $\pm \infty$. Using variational tools we show that the problem has at least three nontrivial smooth solutions (one positive, one negative and a third nodal). Our formulation unifies problems driven by the $p$-Laplacian, the $(p, q)$ Laplacian and the $p$-generalized mean curvature operator.
\end{abstract}

\section{Introduction}

Let $\Omega \subset \mathbb{R}^{N}$ be a bounded domain with a $C^{2}$-boundary $\partial \Omega$. In this paper we study the following nonlinear Neumann problem

$$
-\operatorname{div} a(D u(z))=f(z, u(z)) \quad \text { in } \Omega, \quad \frac{\partial u}{\partial n}=0 \quad \text { on } \partial \Omega .
$$

Here $n(\cdot)$ denotes the outward unit normal on $\partial \Omega$, while $a: \mathbb{R}^{N} \rightarrow \mathbb{R}^{N}$ is a continuous, strictly monotone map which satisfies certain regularity conditions. The precise conditions are formulated in hypotheses $\mathrm{H}(\mathrm{a})$ below. Also, $f: \Omega \times \mathbb{R} \rightarrow \mathbb{R}$ is a Carathéodory reaction (i.e. for all $x \in \mathbb{R}, z \rightarrow f(z, x)$ is measurable and for almost all $z \in \Omega, x \rightarrow f(z, x)$ is continuous) and $x \rightarrow f(z, x)$ exhibits a $(p-1)$ superlinear growth near $\pm \infty$. The hypotheses on $a(\cdot)$ include as a special case

2010 Mathematics Subject Classification. 35J20, 35J60, 35J92.

Key words and phrases. Nonlinear regularity, nonhomogeneous differential operator, unique solution of constant sign, extremal solutions of constant sign, nodal solution. 
the $p$-Laplace differential operator. In this particular case, the asymptotic condition on $f(z, \cdot)$ implies that the equation is resonant with respect to the principal eigenvalue $\widehat{\lambda}_{0}=0$ of the negative Neumann $p$-Laplacian.

The aim of this work is to prove a "three solution theorem" for problem (1.1) and provide precise sign information for all of the solutions. More precisely, we show that problem (1.1) has at least three nontrivial smooth solutions, two of constant sign (one positive and the other negative) and a third one which is nodal (i.e. sign changing). Recently, Aizicovici, Papageorgiou, Staicu [2] and Motreanu, Motreanu, Papageorgiou [17] produced nodal solutions for Neumann equations driven by the $p$-Laplacian with a $(p-1)$-superlinear reaction. Multiplicity results for equations with $(p-1)$-sublinear reaction were proved by Liu [15], Liu and $\mathrm{Su}$ [14], Li and Zhou [12] and Papageorgiou and Papageorgiou [19]. However, all the aforementioned papers deal with equations driven by the Dirichlet $p$ Laplacian and do not produce nodal solutions.

We stress that the differential operator in (1.1) is nonhomogeneous and this is the source of difficulties in the search of a nodal solution. In fact, some of techniques used in [2] and [17] fail here due to the lack of homogeneity and so, different methods and tools are needed to deal with the present setting. Also, the hypotheses on $a(\cdot)$ provide a unified treatment of problems with some important classes of differential operators, such as the $p$-Laplacian, the $(p, q)$-Laplacian and the $p$-generalized mean curvature differential operator.

Our approach is variational, based on the critical point theory. For easy reference, in the next section we present the main mathematical tools that will be used in this paper. We also state the hypotheses on $y \rightarrow a(y)$ and $(z, x) \rightarrow f(z, x)$ and explore some useful consequences of them.

\section{Mathematical background. Hypotheses}

In what follows, by $\|\cdot\|_{p}$ we denote the norm of $L^{p}(\Omega)$ or $L^{p}\left(\Omega, \mathbb{R}^{N}\right)$ and by $\|\cdot\|$ we denote the norm of the Sobolev space $W^{1, p}(\Omega)$, i.e. $\|u\|=\left(\|u\|_{p}^{p}+\right.$ $\left.\|D u\|_{p}^{p}\right)^{1 / p}$ for all $u \in W^{1, p}(\Omega)$. We mention that the notation $\|\cdot\|$ will be also used to denote the $\mathbb{R}^{N}$-norm. No confusion is possible, since it will always be clear from the context which norm we use. The inner product in $\mathbb{R}^{N}$ will be denoted by $(\cdot, \cdot)_{\mathbb{R}^{N}}$.

In addition to the Sobolev space $W^{1, p}(\Omega)$, we will also use the Banach space $C^{1}(\bar{\Omega})$. This is an ordered Banach space with positive cone

$$
C_{+}=\left\{u \in C^{1}(\bar{\Omega}): u(z) \geq 0 \text { for all } z \in \bar{\Omega}\right\} .
$$

This cone has a nonempty interior, given by

$$
\operatorname{int} C_{+}=\left\{u \in C_{+}: u(z)>0 \text { for all } z \in \bar{\Omega}\right\} \text {. }
$$


For $x \in \mathbb{R}$, we define $x^{ \pm}:=\max \{ \pm x, 0\}$ and for $u \in W^{1, p}(\Omega)$ we set $u^{ \pm}(\cdot)=$ $u(\cdot)^{ \pm}$. We have $u^{ \pm} \in W^{1, p}(\Omega)$ and

$$
u=u^{+}-u^{-}, \quad\|u\|=u^{+}+u^{-} .
$$

By $\|\cdot\|_{N}$ we denote the Lebesgue measure on $\mathbb{R}^{N}$. Finally, if $h: \Omega \times \mathbb{R} \rightarrow R$ is a measurable function (for example, a Carathéodory function), then we define

$$
N_{h}(u)(\cdot)=h(\cdot, u(\cdot)) \quad \text { for all } u \in W^{1, p}(\Omega) .
$$

Let $(X,\|\cdot\|)$ be a Banach space and $X^{*}$ its topological dual. By $\langle\cdot, \cdot\rangle$ we denote the duality brackets for the pair $\left(X^{*}, X\right)$. Also, by $\stackrel{w}{\longrightarrow}$ we will designate the weak convergence in $X$.

Given $\varphi \in C^{1}(X)$, we say that $\varphi$ satisfies the Palais-Smale condition (PS-condition, for short), if the following is true:

- every sequence $\left\{x_{n}\right\}_{n \geq 1} \subseteq X$ such that $\left\{\varphi\left(x_{n}\right)\right\}_{n \geq 1} \subseteq \mathbb{R}$ is bounded and

$$
\varphi^{\prime}\left(x_{n}\right) \rightarrow 0 \quad \text { in } X^{*} \text { as } n \rightarrow \infty
$$

admits a strongly convergent subsequence.

This compactness-type condition compensates for the fact that the ambient space is not locally compact and leads to the minimax theorems of critical points theory. In particular, here we will use the following result, known in the literature as the "mountain pass theorem".

TheOREM 2.1. If $\varphi \in C^{1}(X)$ satisfies the PS-condition, $x_{0}, x_{1} \in X$ and $\rho>0$ are such that $\left\|x_{1}-x_{0}\right\|>\rho, \max \left\{\varphi\left(x_{0}\right), \varphi\left(x_{1}\right)\right\}<\inf \left\{\varphi(x):\left\|x-x_{0}\right\|=\rho\right\}$ $=: \eta_{\rho}$, and $c=\inf _{\gamma \in \Gamma} \max _{t \in[0,1]} \varphi(\gamma(t))$, where $\Gamma=\left\{\gamma \in C([0,1], X): \gamma(0)=x_{0}\right.$, $\left.\gamma(1)=x_{1}\right\}$, then $c \geq \eta_{\rho}$ and $c$ is a critical value of $\varphi$ (i.e. there exists $x^{*} \in X$ such that $\varphi^{\prime}\left(x^{*}\right)=0$ and $\left.\varphi\left(x^{*}\right)=c\right)$.

Definition 2.2. A map $A: X \rightarrow X^{*}$ is said to be of type $(\mathrm{S})_{+}$, if for every sequence $\left\{u_{n}\right\}_{n \geq 1} \subseteq X$ such that $u_{n} \stackrel{w}{\longrightarrow} u$ in $X$ and

$$
\lim \sup _{n \rightarrow \infty}\left\langle A\left(u_{n}\right), u_{n}-u\right\rangle \leq 0,
$$

one has $u_{n} \rightarrow u$ in $X$ as $n \rightarrow \infty$.

Near the end of our proof, in order to guarantee the nontriviality of the third solution we will use critical groups. So, let us recall their definition, First of all, given $\varphi \in C^{1}(X)$ and $c \in \mathbb{R}$ we introduce the following sets:

$$
\begin{aligned}
\varphi^{c} & =\{x \in X: \varphi(x) \leq c\}, \\
K_{\varphi} & =\left\{x \in X: \varphi^{\prime}(x)=0\right\}, \\
K_{\varphi}^{c} & =\left\{x \in K_{\varphi}: \varphi(x)=c\right\} .
\end{aligned}
$$


For $Y_{2} \subseteq Y_{1} \subseteq X$ and for every integer $k \geq 0$, by $H_{k}\left(Y_{2}, Y_{1}\right)$ we denote the $k^{\text {th }}$-relative singular homology group for the pair $\left(Y_{1}, Y_{2}\right)$ with integer coefficients. The critical groups of $\varphi$ at an isolated critical point $x \in K_{\varphi}^{c}$ are defined by

$$
C_{k}(\varphi, x)=H_{k}\left(\varphi^{c} \cap U,\left(\varphi^{c} \cap U\right) \backslash\{x\}\right) \quad \text { for all } k \geq 0,
$$

where $U$ is a neighbourhood of $x$ such that $K_{\varphi} \cap \varphi^{c} \cap U=\{x\}$. The excision property of singular homology implies that the above definition of critical groups is independent of the particular choice of the neighbourhood $U$.

Now, let $h \in C^{1}((0, \infty),(0, \infty))$ and assume that

$$
0<\frac{t h^{\prime}(t)}{h(t)} \leq C_{0} \text { for all } t>0, \text { for some } C_{0}>0,
$$

and

$$
C_{1} t^{p-1} \leq h(t) \leq C_{2}\left(1+t^{p-1}\right) \quad \text { for all } t>0 \text {, for some } C_{1}, C_{2}>0,
$$

where $1<p<\infty$. The hypotheses on the map $y \rightarrow a(y)$ are the following:

$\mathrm{H}(\mathrm{a})$ For all $y \in \mathbb{R}^{N}$, one has $a(y)=a_{0}(\|y\|) y, a(0)=0$, with

(i) $a_{0} \in C^{1}((0, \infty),(0, \infty)) . t \rightarrow a_{0}(t) t$ is strictly increasing,

$$
\lim _{t \rightarrow 0^{+}} \frac{t a_{0}^{\prime}(t)}{a_{0}(t)}=C>-1 ;
$$

(ii) for every $y \in \mathbb{R}^{N} \backslash\{0\}$ we have

$$
\|\nabla a(y)\| \leq C_{3} \frac{h(\|y\|)}{\|y\|} \quad \text { for some } C_{3}>0 ;
$$

(iii) for every $y \in \mathbb{R}^{N} \backslash\{0\}$ we have

$$
(\nabla a(y) \xi, \xi)_{\mathbb{R}^{N}} \geq \frac{h(\|y\|)}{\|y\|}\|\xi\|^{2} \quad \text { for all } \xi \in \mathbb{R}^{N} ;
$$

(iv) if $G_{0}(t)=\int_{0}^{t} s a_{0}(s) d s$, then there exist $\tau, \mu \in(1, p)$ such that $t \rightarrow$ $G_{0}\left(t^{1 / \tau}\right)$ is convex on $(0,+\infty)$ and

$$
\lim _{t \rightarrow 0^{+}} \frac{G_{0}(t)}{t^{\mu}}=0 .
$$

REMARKS 2.3. Evidently, $G_{0}$ is strictly convex and strictly increasing. Let

$$
G(y)=G_{0}(\|y\|) \quad \text { for all } y \in \mathbb{R}^{N} .
$$

Then $G$ is convex, $G(0)=0$ and for all $y \in \mathbb{R}^{N} \backslash\{0\}$ we have

$$
\nabla G(y)=G_{0}^{\prime}(\|y\|) \frac{y}{\|y\|}=a_{0}(\|y\|) y=a(y) .
$$


Hence $G(y)$ is the primitive of $a(y)$. The convexity of $G(\cdot)$ implies that

$$
G(y) \leq(a(y), y)_{\mathbb{R}^{N}} \quad \text { for all } y \in \mathbb{R}^{N} .
$$

Using hypotheses $\mathrm{H}(\mathrm{a})$ and (2.1) and (2.2) we infer the following properties of the map $a(\cdot)$.

LEMMA 2.4. If hypotheses $\mathrm{H}(\mathrm{a})$ hold then:

(a) $y \rightarrow a(y)$ is maximal monotone and strictly monotone;

(b) for all $y \in \mathbb{R}^{N}$ we have $\|a(y)\| \leq C_{4}\left(1+\|y\|^{p-1}\right)$ for some $C_{4}>0$;

(c) for all $y \in \mathbb{R}^{N}$ we have $(a(y), y)_{\mathbb{R}^{N}} \geq\left(C_{1} /(p-1)\right)\|y\|^{p}($ see $(2.1))$.

Using this lemma and the integral form of the mean value theorem, we obtain the following growth estimates for the primitive $G(\cdot)$.

Corollary 2.5. If hypotheses $\mathrm{H}(\mathrm{a})$ hold then

$$
\frac{C_{1}}{p(p-1)}\|y\|^{p} \leq G(y) \leq C_{5}\left(1+\|y\|^{p}\right)
$$

for some $C_{5}>0$, all $y \in \mathbb{R}^{N}$.

ExAMPLES 2.6. The following maps satisfy hypotheses $\mathrm{H}(\mathrm{a})$ :

(a) $a(y)=\|y\|^{p-2} y$ with $1<p<\infty$.

This map corresponds to the $p$-Laplace differential operator

$$
u \rightarrow \triangle_{p} u=\operatorname{div}\left(\|D u\|_{\mathbb{R}^{N}}^{p-2} D u\right), \quad \text { for all } u \in W^{1, p}(\Omega) .
$$

(b) $a(y)=\|y\|^{p-2} y+\|y\|^{q-2} y$ with $2 \leq q<p<\infty$.

This map corresponds to the $(p, q)$-Laplace differential operator

$$
u \rightarrow \triangle_{p} u+\triangle_{q} u \text { for all } u \in W^{1, p}(\Omega),
$$

which arises in quantum physics (see Benci, Fortunato and Pisani [3]).

(c) $a(y)=\left(1+\|y\|^{2}\right)^{(p-2) / 2} y$ with $2 \leq p<\infty$.

This map corresponds to the $p$-generalized mean curvature operator

$$
u \rightarrow \operatorname{div}\left(\left(1+\|D u\|^{2}\right)^{(p-2) / 2} D u\right) \quad \text { for all } u \in W^{1, p}(\Omega)
$$

(see Chen-Shen [5]).

(d) $a(y)=\|y\|^{p-2} y+\|y\|^{p-2} y /\left(1+\|y\|^{p}\right)$ with $1<p<\infty$.

Let $f_{0}: \Omega \times \mathbb{R} \rightarrow \mathbb{R}$ be a Carathéodory function with subcritical growth in $x \in \mathbb{R}$, i.e.

$$
\left\|f_{0}(z, x)\right\| \leq \widehat{a}(z)+\widehat{C}\|x\|^{r-1} \quad \text { for a.a. } z \in \Omega \text {, all } x \in \mathbb{R}
$$


with $\widehat{a} \in L^{\infty}(\Omega)_{+}, \widehat{C}>0$ and $1<r<p^{*}$, where

$$
p^{*}= \begin{cases}\frac{N p}{N-p} & \text { if } p<N \\ +\infty & \text { if } N \leq p .\end{cases}
$$

Let $F_{0}(z, x)=\int_{0}^{x} f_{0}(z, s) d s$ and consider the $C^{1}$-functional $\varphi_{0}: W^{1, p}(\Omega) \rightarrow \mathbb{R}$ defined by

$$
\varphi_{0}(u)=\int_{\Omega} G(D u(z)) d z-\int_{\Omega} F_{0}(z, u(z)) d z \quad \text { for all } u \in W^{1, p}(\Omega) .
$$

From Motreanu and Papageorgiou [18], we have the following result.

Proposition 2.7. If $u_{0} \in W^{1, p}(\Omega)$ is a local $C^{1}(\bar{\Omega})$-minimizer of $\varphi_{0}$, i.e. there exists $\rho_{0}>0$ such that

$$
\varphi_{0}\left(u_{0}\right) \leq \varphi_{0}\left(u_{0}+h\right) \quad \text { for all } h \in C^{1}(\bar{\Omega}) \text { with }\|h\|_{C^{1}(\bar{\Omega})} \leq \rho_{0},
$$

then $u_{0} \in C^{1, \alpha}(\bar{\Omega})$ with $\alpha \in(0,1)$ and $u_{0}$ is also a local $W^{1, p}(\Omega)$-minimizer of $\varphi_{0}$, i.e. there exists $\rho_{1}>0$ such that

$$
\varphi_{0}\left(u_{0}\right) \leq \varphi_{0}\left(u_{0}+h\right) \quad \text { for all } h \in W^{1, p}(\Omega) \text { with }\|h\| \leq \rho_{1} .
$$

Remark 2.8. The result of Motreanu-Papageorgiou [18] is stated for a map $a(z, y)$ (i.e. it is also $z$-dependent), which satisfies more restrictive hypotheses in the $y \in \mathbb{R}^{N}$ variable. Nevertheless, a careful inspection of their proof, reveals that it remains valid (in fact verbatim) if we use instead the regularity result of Lieberman ([13, p. 320]).

Let $A: W^{1, p}(\Omega) \rightarrow W^{1, p}(\Omega)^{*}$ be the nonlinear map defined by

$$
\langle A(u), y\rangle=\int_{\Omega}(a(D u), D y)_{\mathbb{R}^{N}} d z \quad \text { for all } u, y \in W^{1, p}(\Omega) .
$$

The next result is a special case of Proposition 2 of Papageorgiou, Rocha and Staicu [20]:

Proposition 2.9. If hypotheses $\mathrm{H}(\mathrm{a})$ hold and $A: W^{1, p}(\Omega) \rightarrow W^{1, p}(\Omega)^{*}$ is the nonlinear map defined by (2.3), then $A$ is maximal monotone and of type $\mathrm{S}_{+}$.

Next we introduce the hypotheses on he reaction $f(t, x)$.

$\mathrm{H}(\mathrm{f}) f: \Omega \times \mathbb{R} \rightarrow \mathbb{R}$ is a Carathéodory function such that $f(z, 0)=0$ for almost all $z \in \Omega$ and

(i) $\|f(z, x)\| \leq \alpha(z)+c\|x\|^{p-1}$ for almost all $z \in \Omega$, all $x \in \mathbb{R}$, with $\alpha \in L^{\infty}(\Omega)_{+}, c>0$

(ii) if $F(z, x)=\int_{0}^{x} f(z, s) d s$, then $\lim _{x \rightarrow \pm \infty} F(z, x) /\|x\|^{p}=0$ uniformly for almost all $z \in \Omega$ and $\lim _{x \rightarrow \pm \infty}[f(z, x) x-p F(z, x)]=+\infty \quad$ uniformly for almost all $z \in \Omega$; 
(iii) there exist $q \in(1, \min \{\mu, \tau\})$ (see $\mathrm{H}(\mathrm{a})(\mathrm{iv})), \theta>q$ with $\theta \leq p^{*}$ if $p<N$ and $\theta<\infty$ if $p \geq N$, and $\delta_{0}>0$ such that $f(z, x) x \geq \widehat{C}_{0}\|x\|^{q}-\widehat{C}_{1}\|x\|^{\theta}$ for almost all $z \in \Omega$, all $x \in \mathbb{R}$, with $\widehat{C}_{0}, \widehat{C}_{1}>0, q F(z, x) \geq f(z, x) x>0$ for almost all $z \in \Omega$, all $\|x\| \leq \delta_{0}$ and ess $\inf F\left(\cdot, \delta_{0}\right)>0$;

(iv) for every $\rho>0$, there exists $\xi_{\rho}>0$ such that $f(z, x) x+\xi_{\rho}\|x\|^{p} \geq 0$ for almost all $z \in \Omega$, all $x \in[-\rho, \rho]$.

REMARK 2.10. In fact it is easy to see that in hypothesis $\mathrm{H}(\mathrm{f})(\mathrm{iii})$, without any loss of generality, we can always assume that $\theta \geq p$.

EXAmPle 2.11. The following function satisfies hypotheses $\mathrm{H}(\mathrm{f})$ (for the sake of simplicity we drop the $z$-dependence):

$$
f(x)=\|x\|^{q-2} x-\|x\|^{r-2} x \text { for all } x \in \mathbb{R} \text {, with } 1<q<r<p .
$$

\section{Constant sign solutions}

In this section we produce two smooth solutions of constant sign (one positive, the other negative). To this end, we introduce the following truncationperturbations of $f(z, \cdot)$ :

$$
\begin{array}{ll}
\widehat{f}_{+}(z, x)=f\left(z, x^{+}\right)+\left(x^{+}\right)^{p-1} & \text { for all } x \in \mathbb{R}, \\
\widehat{f}_{-}(z, x)=f\left(z,-x^{-}\right)-\|x\|^{p-2} x^{-} & \text {for all } x \in \mathbb{R} .
\end{array}
$$

Both are Carathéodory functions. We set $\widehat{F}_{ \pm}(z, x)=\int_{0}^{x} \widehat{f}_{ \pm}(z, s) d s$ and consider the $C^{1}$-functionals $\widehat{\varphi}_{ \pm}:: W^{1, p}(\Omega) \rightarrow \mathbb{R}$ defined by

$$
\widehat{\varphi}_{ \pm}(u)=\int_{\Omega} G(D u(z)) d z+\frac{1}{p}\|u\|_{p}^{p}-\int_{\Omega} \widehat{F}_{ \pm}(z, u(z)) d z \quad \text { for all } u \in W^{1, p}(\Omega) .
$$

Proposition 3.1. If hypotheses $\mathrm{H}(\mathrm{a})$ and $\mathrm{H}(\mathrm{f})$ hold, then problem (1.1) has at least two nontrivial constant sign smooth solutions $u_{0} \in \operatorname{int} C_{+}$and $v_{0} \in$ $-\operatorname{int} C_{+}$.

Proof. First we produce the nontrivial positive solution. By virtue of hypothesis $\mathrm{H}(\mathrm{f})(\mathrm{ii})$, given $\xi>0$, we can find $M_{1}=M_{1}(\xi)>0$, such that

$$
f(z, x) x-p F(z, x) \geq \xi \text { for a.a. } z \in \Omega \text {, all } x \geq M_{1} .
$$

Then, by (3.1), for almost all $z \in \Omega$ and all $x \geq M_{1}$, we have

$$
\frac{d}{d x}\left(\frac{F(z, x)}{x^{p}}\right)=\frac{f(z, x) x-p F(z, x)}{x^{p+1}} \geq \frac{\xi}{x^{p+1}}
$$

hence

$$
\frac{F(z, x)}{x^{p}}-\frac{F(z, y)}{y^{p}} \geq-\frac{\xi}{p}\left(\frac{1}{x^{p}}-\frac{1}{y^{p}}\right) \quad \text { for a.a. } z \in \Omega, \text { all } x \geq y \geq M_{1} .
$$


Passing to the limit as $x \rightarrow \infty$ and using hypothesis $\mathrm{H}(\mathrm{f})(\mathrm{ii})$, we have

$$
-\frac{F(z, y)}{y^{p}} \geq \frac{\xi}{p y^{p}} \quad \text { for a.a. } z \in \Omega, \text { all } y \geq M_{1},
$$

hence

$$
F(z, y) \leq-\frac{\xi}{p} \text { for a.a. } z \in \Omega, \text { all } y \geq M_{1}
$$

Since $\xi>0$ is arbitrary, we infer that

$$
F(z, y) \rightarrow-\infty \quad \text { uniformly for a.a. } z \in \Omega \text {, as } x \rightarrow+\infty \text {. }
$$

Claim. $\widehat{\varphi}_{+}$is coercive.

Suppose that the Claim is not true. Then we can find $\left\{u_{n}\right\}_{n \geq 1} \subset W^{1, p}(\Omega)$ such that

$$
\left\|u_{n}\right\| \rightarrow \infty \text { and } \widehat{\varphi}_{+}\left(u_{n}\right) \leq M_{2} \text { for some } M_{2}>0 \text {, all } n \geq 1 .
$$

Let $y_{n}=u_{n} /\left\|u_{n}\right\|$, for $n \geq 1$. Then $\left\|y_{n}\right\|=1$ for all $n \geq 1$ and so, by passing to a suitable subsequence if necessary, we may assume that

$$
y_{n} \stackrel{w}{\longrightarrow} y \quad \text { in } W^{1, p}(\Omega) \quad \text { and } \quad y_{n} \rightarrow y \quad \text { in } L^{p}(\Omega) \text { as } n \rightarrow \infty .
$$

From (3.3) and Corollary 2.5, we have

$$
\frac{C_{1}}{p(p-1)}\left\|D u_{n}\right\|_{p}^{p}+\frac{1}{p}\left\|u_{n}\right\|_{p}^{p}-\int_{\Omega} \widehat{F}_{+}\left(z, u_{n}\right) d z \leq M_{2}, \quad \text { for all } n \geq 1 .
$$

hence, by the definition of $\widehat{F}_{+}$,

$$
\frac{C_{1}}{p(p-1)}\left\|D u_{n}\right\|_{p}^{p}+\frac{1}{p}\left\|u_{n}\right\|_{p}^{p}-\int_{\Omega} F\left(z, u_{n}^{+}\right) d z-\frac{1}{p}\left\|u_{n}^{+}\right\|_{p}^{p} \leq M_{2},
$$

therefore

$$
\begin{aligned}
\frac{C_{1}}{p(p-1)}\left\|D u_{n}\right\|_{p}^{p}+\frac{1}{p}\left\|u_{n}^{-}\right\|_{p}^{p}-\int_{\left\{u_{n} \geq M_{1}\right\}} F\left(z, u_{n}^{+}\right) d z & -\int_{\left\{0 \leq u_{n}<M_{1}\right\}} F\left(z, u_{n}^{+}\right) d z \leq M_{2},
\end{aligned}
$$

where $M_{1}>0$ is as in (3.1). By (3.3), we have

$$
-\int_{\left\{u_{n} \geq M_{1}\right\}} F\left(z, u_{n}\right) d z \geq 0 .
$$

Also, by $\mathrm{H}(\mathrm{f})(\mathrm{i})$,

$$
\int_{\left\{0 \leq u_{n}<M_{1}\right\}} F\left(z, u_{n}\right) d z \leq \widehat{C}, \quad \text { for some } \widehat{C}>0 .
$$

Using (3.6) and (3.7) in (3.5), we arrive at

$$
\frac{C_{1}}{p(p-1)}\left\|D u_{n}\right\|_{p}^{p}+\frac{1}{p}\left\|u_{n}^{-}\right\|_{p}^{p} \leq M_{2}^{\prime},
$$


with $M_{2}^{\prime}=M_{2}^{\prime}+\widehat{C}$. This yields

$$
\frac{C_{1}}{p(p-1)}\left\|D y_{n}\right\|_{p}^{p}+\frac{1}{p}\left\|y_{n}^{-}\right\|_{p}^{p} \leq \frac{M_{2}^{\prime}}{\left\|u_{n}\right\|^{p}} .
$$

So, if we pass to the limit as $n \rightarrow \infty$ in (3.8) and use (3.4), then

$$
\frac{C_{1}}{p(p-1)}\|D y\|_{p}^{p}+\frac{1}{p}\left\|y^{-}\right\|_{p}^{p} \leq 0
$$

hence $y=\xi \in \mathbb{R}^{+}$. Then $D y_{n} \rightarrow 0=D \xi$ in $L^{p}\left(\Omega, \mathbb{R}^{N}\right)$ and $y_{n} \rightarrow \xi$ in $L^{p}(\Omega)$, hence $y_{n} \rightarrow \xi$ in $W^{1, p}(\Omega)$ and so, $\|\xi\|=1$, i.e. $\xi \neq 0$. Therefore $\xi>0$ and so we infer that $u_{n}(z) \rightarrow \infty$ for almost all $z \in \Omega$. Then, from the first part of the proof it follows that $\widehat{F}_{+}\left(z, u_{n}(z)\right) \rightarrow-\infty$ for almost all $z \in \Omega$, hence

$$
\int_{\Omega} \widehat{F}_{+}\left(z, u_{n}(z)\right) d z \rightarrow-\infty
$$

(by Fatou's lemma). But from (3.3) we have

$$
\int_{\Omega} \widehat{F}_{+}\left(z, u_{n}(z)\right) \geq-M_{2} \text { for all } n \geq 1,
$$

a contradiction. This proves the Claim.

Using the Sobolev embedding theorem, we can easily see that $\widehat{\varphi}_{+}$is sequentially weakly lower semicontinuous. So, by the Weierstrass theorem, we can find $u_{0} \in W^{1, p}(\Omega)$ such that

$$
\widehat{\varphi}_{+}\left(u_{0}\right)=\inf \left\{\widehat{\varphi}_{+}(u): u \in W^{1, p}(\Omega)\right\}=\widehat{m}_{+} .
$$

By virtue of hypothesis $\mathrm{H}(\mathrm{a})(\mathrm{iv})$, we can find $\delta>0$ such that

$$
G(y) \leq\|y\|^{\mu} \quad \text { for all } y \in \mathbb{R}^{N} \text { with }\|y\| \leq \delta .
$$

Let $u \in C_{+} \backslash\{0\}$ and let $\lambda \in(0,1)$ be small, such that $\lambda D u(z) \in[0, \delta]$ for all $z \in \bar{\Omega}$. Then

$$
\begin{array}{rlrl}
\widehat{\varphi}_{+}(\lambda u) & =\int_{\Omega} G(\lambda D u) d z-\int_{\Omega} F(z, \lambda u) d z & & (\text { since } u \geq 0) \\
& \leq \lambda^{\mu}\|D u\|_{\mu}^{\mu}+\frac{\widehat{C}_{1}}{\theta} \lambda^{\theta}\|u\|_{\theta}^{\theta}-\frac{\widehat{C}_{0}}{q} \lambda^{q}\|u\|_{q}^{q} & & (\text { see }(3.10) \\
& & \text { and H(f)(iii)). }
\end{array}
$$

Because $1<q<\mu, \theta$ by (3.11) and by choosing $\lambda \in(0,1)$ even smaller if necessary, we obtain $\widehat{\varphi}_{+}(\lambda u)<0$, hence $\widehat{\varphi}_{+}\left(u_{0}\right)=\widehat{m}_{+}<0=\widehat{\varphi}_{+}(0)$ (see (3.9)), therefore $u_{0} \neq 0$. From (3.9) we have $\widehat{\varphi}_{+}^{\prime}\left(u_{0}\right)=0$, hence

$$
A\left(u_{0}\right)+\left\|u_{0}\right\|^{p-2} u_{0}=N_{\widehat{f}_{+}}\left(u_{0}\right) .
$$


On $(3.12)$ we act with $-u_{0}^{-} \in W^{1, p}(\Omega)$ and obtain

$$
\int_{\Omega}\left(a\left(D u_{0}\right),-D u_{0}^{-}\right)_{\mathbb{R}^{N}} d z+\left\|u_{0}^{-}\right\|_{p}^{p}=0
$$

hence

$$
\frac{C_{1}}{p-1}\left\|D u_{0}^{-}\right\|_{p}^{p}+\left\|u_{0}^{-}\right\|_{p}^{p} \leq 0
$$

(see Lemma 2.4(c)), therefore $u_{0} \geq 0, u_{0} \neq 0$.

From (3.12) and the nonlinear Green's identity (see for example, Gasinski and Papageorgiou [8, p. 210]), we have

$$
-\operatorname{div} a\left(D u_{0}(z)\right)=f\left(z, u_{0}(z)\right) \quad \text { a.e. in } \Omega, \quad \frac{\partial u_{0}}{\partial n}=0 \quad \text { on } \partial \Omega .
$$

From Gasinski and Papageorgiou [9] (Proposition 3.1), it follows that $u_{0} \in$ $L^{\infty}(\Omega)$. Then the regularity result of Lieberman $([13$, p. 320]) implies that $u_{0} \in C_{+} \backslash\{0\}$.

Let $\rho=\left\|u_{0}\right\|_{\infty}$ and let $\xi_{\rho}$ be as postulated by $\mathrm{H}(\mathrm{f})(\mathrm{iv})$. Then

$-\operatorname{div} a\left(D u_{0}(z)\right)+\xi_{\rho} u_{0}(z)^{p-1}=f\left(z, u_{0}(z)\right)+\xi_{\rho} u_{0}(z)^{p-1} \geq 0 \quad$ a.e. in $\Omega$, hence

$$
\operatorname{div} a\left(D u_{0}(z)\right) \leq \xi_{\rho} u_{0}(z)^{p-1} \quad \text { a.e. in } \Omega .
$$

Invoking Theorem 5.5.1 of Pucci and Serrin ([21, p. 120]) we conclude that $u_{0} \in \operatorname{int} C_{+}$.

Similarly, working with $\widehat{\varphi}_{-}$(which too is coercive, since $F(z, x) \rightarrow-\infty$ uniformly for almost all $z \in \Omega$, as $x \rightarrow-\infty)$, we obtain a second nontrivial constant sign smooth solution $v_{0} \in-\operatorname{int} C_{+}$.

\section{A nodal solution}

In this section, we produce a third nontrivial smooth solution which is nodal. To this end, we consider the following auxiliary nonlinear Neumann problem

$$
\begin{aligned}
-\operatorname{div} a(D u(z)) & =\widehat{C}_{0}\|u(z)\|^{q-2} u(z)-\widehat{C}_{1}\|u(z)\|^{\theta-2} u(z) & & \text { in } \Omega, \\
\frac{\partial u}{\partial n} & =0 & & \text { on } \partial \Omega,
\end{aligned}
$$

where $\widehat{C}_{0}, \widehat{C}_{1}$ are as in hypothesis $\mathrm{H}(\mathrm{f})(\mathrm{iii})$.

Proposition 4.1. If hypotheses $\mathrm{H}(\mathrm{a})$ hold, then problem (4.1) has a unique nontrivial positive solution $u_{*} \in$ int $C_{+}$and a unique nontrivial negative solution $v_{*}=-u_{*} \in-\operatorname{int} C_{+}$.

Proof. First we establish the existence of a nontrivial positive solution. So, let $\psi_{+}: W^{1, p}(\Omega) \rightarrow \mathbb{R}$ be the $C^{1}$-functional defined by

$$
\psi_{+}(u)=\int_{\Omega} G(D u) d z+\frac{1}{p}\|u\|_{p}^{p}-\frac{\widehat{C}_{0}}{q}\left\|u^{+}\right\|_{q}^{q}+\frac{\widehat{C}_{1}}{\theta}\left\|u^{+}\right\|_{\theta}^{\theta}-\frac{1}{p}\left\|u^{+}\right\|_{p}^{p},
$$


for all $u \in W^{1, p}(\Omega)$. Then

$\psi_{+}(u) \geq \frac{C_{1}}{p(p-1)}\left\|D u_{0}\right\|_{p}^{p}+\frac{1}{p}\left\|u^{-}\right\|_{p}^{p}+\frac{\widehat{C}_{1}}{\theta}\left\|u^{+}\right\|_{\theta}^{\theta}-\frac{\widehat{C}_{0}}{q}\left\|u^{+}\right\|_{q}^{q} \geq C_{6}\|u\|^{p}-C_{7}\|u\|^{q}$ for some $C_{6}, C_{7}>0$ (see [8, p. 227]), therefore $\psi_{+}$is coercive (recall that $p>q$ ). Also, using the Sobolev embedding theorem, we can show that $\psi_{+}$is sequentially weakly lower semicontinuous. Therefore we can find $u_{*} \in W^{1, p}(\Omega)$ such that

$$
\psi_{+}\left(u_{*}\right)=\inf \left\{\psi_{+}(u): u \in W^{1, p}(\Omega)\right\}=m_{+}^{*} .
$$

As in the proof of Proposition 3.1, using hypotheses $\mathrm{H}(\mathrm{a})(\mathrm{iv}), \mathrm{H}(\mathrm{f})$ (iii) and the fact that $q<\mu, \theta$, we show that

$$
\psi_{+}\left(u_{*}\right)=m_{+}^{*}<0=\psi_{+}(0),
$$

i.e. $u_{*} \neq 0$. From $(4.2)$, we have $\psi_{+}^{\prime}\left(u_{*}\right)=0$, hence

$$
A\left(u_{*}\right)+\left\|u_{*}\right\|^{p-2} u_{*}=\widehat{C}_{0}\left(u_{*}^{+}\right)^{q-1}-\widehat{C}_{1}\left(u_{*}^{+}\right)^{\theta-1}+\left(u_{*}^{+}\right)^{p-1} .
$$

Acting on (4.3) with $-u_{*}^{-} \in W^{1, p}(\Omega)$ and using Lemma 2.4(c) we obtain

$$
\frac{C_{1}}{p-1}\left\|D u_{*}^{-}\right\|_{p}^{p}+\left\|u_{*}^{-}\right\|_{p}^{p} \leq 0
$$

i.e. $u_{*} \geq 0, u_{*} \neq 0$. Then, (4.3) becomes

$$
A\left(u_{*}\right)=\widehat{C}_{0}\left(u_{*}^{+}\right)^{q-1}-\widehat{C}_{1}\left(u_{*}^{+}\right)^{\theta-1},
$$

hence

$$
-\operatorname{div} a\left(D u_{*}(z)\right)=\widehat{C}_{0}\left(u_{*}(z)\right)^{q-1}-\widehat{C}_{1}\left(u_{*}(z)\right)^{\theta-1} \quad \text { a.e. in } \Omega, \frac{\partial u_{*}}{\partial n}=0 \text { on } \partial \Omega .
$$

Nonlinear regularity (see Lieberman [13, p. 320]) and the nonlinear maximum principle (see Pucci and Serrin [21, p. 120]) imply that $u_{*} \in \operatorname{int} C_{+}$.

Next we show the uniqueness of this positive solution. To this end, let $\xi_{+}: L^{1}(\Omega) \rightarrow \overline{\mathbb{R}}=\mathbb{R} \cup\{\infty\}$ be the integral functional defined by

$$
\xi_{+}(u)= \begin{cases}\int_{\Omega} G\left(D u^{1 / \tau}\right) d z & \text { if } u \geq 0, u^{1 / \tau} \in W^{1, p}(\Omega) \\ +\infty & \text { otherwise. }\end{cases}
$$

Let $u_{1}, u_{2} \in \operatorname{dom} \xi_{+}$. For $t \in[0,1]$ we define

$$
y=\left(t u_{1}+(1-t) u_{2}\right)^{1 / \tau} .
$$

We set $v_{1}=u_{1}^{1 / \tau}$ and $v_{2}=u_{2}^{1 / \tau}$. As in Lemma 1 of Diaz and Saa [6] (see also Benguria, Brezis, Lieb [4, Lemma 4]), via Hölder's inequality, we have

$$
\|D y(z)\| \leq\left(t\left\|D v_{1}(z)\right\|^{\tau}+(1-t)\left\|D v_{2}(z)\right\|^{\tau}\right)^{1 / \tau} \quad \text { a.e. in } \Omega .
$$

Since $G_{0}$ is increasing, we obtain

$$
G_{0}(\|D y(z)\|) \leq G_{0}\left(\left(t\left\|D v_{1}(z)\right\|^{\tau}+(1-t)\left\|D v_{2}(z)\right\|^{\tau}\right)^{1 / \tau}\right) \quad \text { a.e. in } \Omega \text {. }
$$


Hypothesis $\mathrm{H}(\mathrm{a})(\mathrm{iv})$ implies that

$$
\begin{aligned}
G_{0}\left(\left(t\left\|D v_{1}(z)\right\|^{\tau}+\right.\right. & \left.\left.(1-t)\left\|D v_{2}(z)\right\|^{\tau}\right)^{1 / \tau}\right) \\
& \leq t G_{0}\left(\left\|D v_{1}(z)\right\|\right)+(1-t) G_{0}\left(\left\|D v_{2}(z)\right\|\right) \quad \text { a.e. in } \Omega
\end{aligned}
$$

Recalling that $G(y)=G_{0}(\|y\|)$ for all $y \in \mathbb{R}^{N}$, from (4.4) and (4.5), we have

$$
G(D y(z)) \leq t G\left(D u_{1}(z)^{1 / \tau}\right)+(1-t) G\left(D u_{2}(z)^{1 / \tau}\right) \quad \text { a.e. in } \Omega
$$

hence $\xi_{+}$is convex. Also, via Fatou's lemma, we infer that $\xi_{+}$is lower semicontinuous and of course $\operatorname{dom} \xi_{+} \neq \emptyset$ (i.e. $\xi_{+} \in \Gamma_{0}\left(L^{1}(\Omega)\right.$ ); see Gasinski and Papageorgiou [8, p. 488]).

Let $u \in W^{1, p}(\Omega)$ be a nontrivial positive solution of (4.1). From the first part of the proof, we know that $u \in \operatorname{int} C_{+}$. Then $u^{\tau} \geq 0,\left(u^{\tau}\right)^{1 / \tau}=u \in W^{1, p}(\Omega)$, hence $u^{\tau} \in \operatorname{dom} \xi_{+}$. Let $h \in C_{+} \backslash\{0\}$. Then for $\lambda \in(-1,1)$ small, we have $u^{\tau}+\lambda h \in \operatorname{int} C_{+}$and so $u^{\tau}+\lambda h \in \operatorname{dom} \xi_{+}$. Hence, the Gateaux derivative of $\xi_{+}$ at $u^{\tau}$ in the direction $h$ exists, and the chain rule implies that

$$
\xi_{+}^{\prime}\left(u^{\tau}\right)(h)=\frac{1}{\tau} \int_{\Omega} \frac{-\operatorname{div} a(D u)}{u^{\tau-1}} h d z .
$$

Similarly, if $v \in W^{1, p}(\Omega)$ is another nontrivial positive solution of (4.1), then $v \in \operatorname{int} C_{+}$and

$$
\xi_{+}^{\prime}\left(v^{\tau}\right)(h)=\frac{1}{\tau} \int_{\Omega} \frac{-\operatorname{div} a(D v)}{v^{\tau-1}} h d z .
$$

The convexity of $\xi_{+}$implies that $\xi_{+}^{\prime}$ is monotone. Hence, from (4.6) and (4.7), we have

$$
0 \leq \int_{\Omega}\left(\frac{-\operatorname{div} a(D u)}{u^{\tau-1}}+\frac{\operatorname{div} a(D v)}{v^{\tau-1}}\right)\left(u^{\tau}-v^{\tau}\right) d z,
$$

hence (cf. (4.1))

$$
0 \leq \int_{\Omega}\left(\widehat{C}_{0}\left(\frac{1}{u^{\tau-q}}-\frac{1}{v^{\tau-q}}\right)-\widehat{C}_{1}\left(u^{\theta-\tau}-v^{\theta-\tau}\right)\right)\left(u^{\tau}-v^{\tau}\right) d z
$$

Since $q<\tau<\theta$ (see H(f)(iii)), the function $x \rightarrow \widehat{C}_{0} / x^{\tau-q}-\widehat{C}_{1} x^{\theta-\tau}$ is strictly decreasing on $(0, \infty)$. So, from (4.8) we infer that $u=v$. This proves the uniqueness of $u_{*} \in \operatorname{int} C_{+}$.

The oddness of (4.1) implies that $v_{*}=-u_{*} \in-\operatorname{int} C_{+}$is the unique nontrivial negative solution of (4.1).

Using this proposition, we can establish the existence of extremal solutions of constant sign, i.e. we show that there exists a smallest nontrivial positive solution and a biggest nontrivial negative solution of (1.1). 
Proposition 4.2. If hypotheses $\mathrm{H}(\mathrm{a})$ and $\mathrm{H}(\mathrm{f})$ hold, then problem (1.1) has a smallest nontrivial positive solution $u_{+} \in \operatorname{int} C_{+}$and a biggest nontrivial negative solution $v_{-} \in-\operatorname{int} C_{+}$.

Proof. Let $\mathcal{S}_{+}$be the set of nontrivial positive solutions of (1.1). From Proposition 3.1 and its proof we know that $\mathcal{S}_{+} \neq \emptyset$ and $\mathcal{S}_{+} \subset \operatorname{int} C_{+}$.

Claim. If $\widetilde{u} \in \mathcal{S}_{+}$then $u_{*} \leq \widetilde{u}$.

Let $\gamma_{+}: \Omega \times \mathbb{R} \rightarrow \mathbb{R}$ be the Carathéodory function defined by

$$
\gamma_{+}(z, x)= \begin{cases}0 & \text { if } x<0, \\ \widehat{C}_{0} x^{q-1}-\widehat{C}_{1} x^{\theta-1}+x^{p-1} & \text { if } 0 \leq x \leq \widetilde{u}(z), \\ \widehat{C}_{0} \widetilde{u}(z)^{q-1}-\widehat{C}_{1} \widetilde{u}(z)^{\theta-1}+\widetilde{u}(z)^{p-1} & \text { if } \widetilde{u}(z)<x\end{cases}
$$

We set

$$
\Gamma_{+}(z, x)=\int_{0}^{x} \gamma_{+}(z, s) d s
$$

and consider the $C^{1}$-functional $\beta_{+}: W^{1, p}(\Omega) \rightarrow \mathbb{R}$ defined by

$$
\beta_{+}(u)=\int_{\Omega} G(D u) d z+\frac{1}{p}\|u\|_{p}^{p}-\int_{\Omega} \Gamma_{+}(z, u(z)) d z \quad \text { for all } u \in W^{1, p}(\Omega) .
$$

From Corollary 2.5 and (4.9), we see that $\beta_{+}$is coercive. Also, it is sequentially weakly lower semicontinuous. So, we can find $\widehat{y} \in W^{1, p}(\Omega)$ such that

$$
\beta_{+}(\widehat{y})=\inf \left\{\beta_{+}(u): u \in W^{1, p}(\Omega)\right\}=\widehat{m}_{+} .
$$

As in the proof of Proposition 3.1, using hypotheses H(a)(iv), H(f)(iii) and recalling that $1<q<\mu, \theta$ we conclude that

$$
\beta_{+}(\widehat{y})=\widehat{m}_{+}<0=\beta_{+}(0) \quad(\text { see }(4.10)), \text { i.e. } \widehat{y} \neq 0 .
$$

From (4.10) we have $\beta_{+}^{\prime}(\widehat{y})=0$, hence

$$
A(\widehat{y})+\|\widehat{y}\|^{p-2} \widehat{y}=N_{\gamma_{+}}(\widehat{y}) .
$$

On (4.11) we act with $-\widehat{y}^{-} \in W^{1, p}(\Omega)$ and use Lemma 2.4(c) to obtain

$$
\frac{C_{1}}{p-1}\left\|D \widehat{y}^{-}\right\|_{p}^{p}+\left\|\widehat{y}^{-}\right\|_{p}^{p} \leq 0
$$

(see (4.9)) hence $\widehat{y} \geq 0, \widehat{y} \neq 0$. 
Acting on (4.11) with $(\widehat{y}-\widetilde{u})^{+} \in W^{1, p}(\Omega)$, we obtain

$$
\begin{aligned}
& \left\langle A(\widehat{y}),(\widehat{y}-\widetilde{u})^{+}\right\rangle+\int_{\Omega} \widehat{y}^{p-1}(\widehat{y}-\widetilde{u})^{+} d z \\
& \quad=\int_{\Omega} \gamma_{+}(z, \widehat{y})(\widehat{y}-\widetilde{u})^{+} d z \\
& \quad=\int_{\Omega}\left(\widehat{C}_{0} \widetilde{u}^{q-1}-\widehat{C}_{1} \widetilde{u}^{\theta-1}\right)(\widehat{y}-\widetilde{u})^{+} d z+\int_{\Omega} \widetilde{u}^{p-1}(\widehat{y}-\widetilde{u})^{+} d z \quad \text { (see (4.9)) } \\
& \leq \int_{\Omega} f(z, \widetilde{u})(\widehat{y}-\widetilde{u})^{+} d z+\int_{\Omega} \widetilde{u}^{p-1}(\widehat{y}-\widetilde{u})^{+} d z \\
& =\left\langle A(\widetilde{u}),(\widehat{y}-\widetilde{u})^{+}\right\rangle+\int_{\Omega} \widetilde{u}^{p-1}(\widehat{y}-\widetilde{u})^{+} d z
\end{aligned}
$$

hence

$$
\int_{\{\widehat{y}>\widetilde{u}\}}(a(D \widehat{y})-a(D \widetilde{u}), D \widehat{y}-D \widetilde{u})_{\mathbb{R}^{N}} d z+\int_{\{\widehat{y}>\widetilde{u}\}}\left(\widehat{y}^{p-1}-\widetilde{u}^{p-1}\right)(\widehat{y}-\widetilde{u}) \leq 0
$$

therefore $\|\{\widehat{y}>\widetilde{u}\}\|_{N}=0$, i.e. $\widehat{y} \leq \widetilde{u}$ (see Lemma 2.4(a)). So, we have proved that

$$
\widehat{y} \in[0, \widetilde{u}]=\left\{u \in W^{1, p}(\Omega): 0 \leq u(z) \leq \widetilde{u}(z)\right\}, \quad y \neq 0 .
$$

Then (4.11) becomes

$$
A(\widehat{y})=\widehat{C}_{0} \widehat{y}^{q-1}-\widehat{C}_{1} \widehat{y}^{\theta-1}
$$

(see (4.9)), hence

$$
-\operatorname{div} a(D \widehat{y}(z))=\widehat{C}_{0} \widehat{y}(z)^{q-1}-\widehat{C}_{1} \widehat{y}(z)^{\theta-1} \quad \text { a.e. in } \Omega, \quad \frac{\partial \widehat{y}}{\partial n}=0 \quad \text { on } \partial \Omega,
$$

and we conclude that $\widehat{y}=u_{*}$ (see Proposition 4.1). Therefore we have $u_{*} \leq \widetilde{u}$ and this proves the Claim.

Now, let $C \subset \mathcal{S}_{+}$be a chain (i.e. a linearly ordered subset of $\mathcal{S}_{+}$). From Dunford and $\operatorname{Schwartz}\left([7\right.$, p. 336] $)$, we know that there exists a sequence $\left\{u_{n}\right\}_{n \geq 1} \subset$ $C$ such that $\inf _{C}=\inf _{n \geq 1} u_{n}$. Moreover, according to Heikkila and Lakshmikantham ([10, p. 15]), we may assume that $\left\{u_{n}\right\}_{n \geq 1}$ is decreasing.

We have

$$
A\left(u_{n}\right)=N_{f}\left(u_{n}\right) \text { and } u_{*} \leq u_{n} \leq u_{1} \text { for all } n \geq 1
$$

(see the Claim), hence $\left\{u_{n}\right\}_{n \geq 1} \subset W^{1, p}(\Omega)$ is bounded. So, we may assume that

$$
u_{n} \stackrel{w}{\longrightarrow} u \quad \text { in } W^{1, p}(\Omega) \text { and } \quad u_{n} \rightarrow u \quad \text { in } L^{p}(\Omega) \quad \text { as } n \rightarrow \infty .
$$

On (4.12) we act with $u_{n}-u \in W^{1, p}(\Omega)$, pass to the limit as $n \rightarrow \infty$ and use (4.13). Then

hence

$$
\lim _{n \rightarrow \infty}\left\langle A\left(u_{n}\right), u_{n}-u\right\rangle=0
$$

$$
u_{n} \rightarrow u \quad \text { in } W^{1, p}(\Omega) \text { as } n \rightarrow \infty
$$


(see Proposition 2.9). So, if in (4.12) we pass to the limit as $n \rightarrow \infty$ and use (4.14), then $A(u)=N_{f}(u)$ and $u_{*} \leq u$ (see (4.12)), hence $u \in \mathcal{S}_{+}$and $u=\inf C$.

Since $C$ is an arbitrary chain in $\mathcal{S}_{+}$, by the Kuratowski-Zorn lemma, it follows that $\mathcal{S}_{+}$has a minimal element $u_{+} \in \mathcal{S}_{+}$.

As in Aizicovici-Papageorgiou-Staicu [2] (see Lemma 1 and the proof of Proposition 8) we show that $\mathcal{S}_{+}$is downward directed (i.e. if $u_{1}, u_{2} \in \mathcal{S}_{+}$, then there exists $u \in \mathcal{S}_{+}$such that $u \leq u_{1}$ and $u \leq u_{2}$ ).

Therefore, $u_{+} \in \mathcal{S}_{+}$is the smallest nontrivial positive solution of (1.1).

Similarly, let $\mathcal{S}_{-}$be the set of nontrivial negative solutions of (1.1). Then $\mathcal{S}_{-} \neq \emptyset, \mathcal{S}_{-} \subset-\operatorname{int} C_{+}$and $\mathcal{S}_{-}$is upward directed (i.e. if $v_{1}, v_{2} \in \mathcal{S}_{-}$, then there exists $v \in \mathcal{S}_{-}$such that $v_{1} \leq v$ and $v_{2} \leq v$ ) (see [2, Lemma 2]). Reasoning as above, we obtain $v_{-} \in-\operatorname{int} C_{+}$, the biggest nontrivial negative solution of (1.1).

REMARK 4.3. The proofs of Propositions 4.1 and 4.2 are somewhat comparable to those of [16], Lemma 2.2 and Lemmas 4.1-4.2, respectively.

Using these extremal solutions, we can establish the existence of a nodal solution for problem (1.1).

Proposition 4.4. If hypotheses $\mathrm{H}(\mathrm{a})$ and $\mathrm{H}(\mathrm{f})$ hold, then problem (1.1) has a nodal solution $y_{0} \in C^{1}(\bar{\Omega})$.

Proof. Let $u_{+} \in \operatorname{int} C_{+}$and $v_{-} \in-\operatorname{int} C_{+}$be the two extremal nontrivial constant sign solutions of (1.1). We consider the following truncation of the reaction $f(z, \cdot)$ :

$$
\widetilde{f}(z, x)= \begin{cases}f\left(z, v_{-}(z)\right)+\left\|v_{-}(z)\right\|^{p-2} v_{-}(z) & \text { if } x<v_{-}(z) \\ f(z, x)+\|x\|^{p-2} x & \text { if } v_{-}(z) \leq x \leq u_{+}(z) \\ f\left(z, u_{+}(z)\right)+u_{+}(z)^{p-1} & \text { if } u_{+}(z)<x\end{cases}
$$

This is a Carathéodory function. We set

$$
\widetilde{F}(z, x)=\int_{0}^{x} \widetilde{f}(z, s) d s
$$

and introduce the $C^{1}$-functional $\widetilde{\xi}: W^{1, p}(\Omega) \rightarrow \mathbb{R}$ defined by

$$
\widetilde{\xi}(u)=\int_{\Omega} G(D u) d z+\frac{1}{p}\|u\|_{p}^{p}-\int_{\Omega} \widetilde{F}(z, u(z)) d z, \quad \text { for all } u \in W^{1, p}(\Omega) .
$$

Also, let

$$
\widetilde{f}_{ \pm}(z, x)=\widetilde{f}\left(z, \pm x^{ \pm}\right), \widetilde{F}_{ \pm}(z, x)=\int_{0}^{x} \widetilde{f}_{ \pm}(z, s) d s
$$

and consider the $C^{1}$-functionals $\widetilde{\xi}_{ \pm}: W^{1, p}(\Omega) \rightarrow \mathbb{R}$ defined by

$$
\widetilde{\xi}_{ \pm}(u)=\int_{\Omega} G(D u) d z-\int_{\Omega} \widetilde{F}_{ \pm}(z, u(z)) d z, \quad \text { for all } u \in W^{1, p}(\Omega) .
$$


As before (see for example, the proof of Proposition 4.2, using (4.15) we show that $K_{\widetilde{\xi}} \subset\left[v_{-}, u_{+}\right], K_{\widetilde{\xi}_{+}} \subset\left[0, u_{+}\right], K_{\widetilde{\xi}_{-}} \subset\left[v_{-}, 0\right]$. The extremality of the solutions $u_{+}$and $v_{-}$implies that

$$
K_{\widetilde{\xi}} \subseteq\left[v_{-}, u_{+}\right], \quad K_{\widetilde{\xi}_{+}}=\left\{0, u_{+}\right\}, \quad K_{\widetilde{\xi}_{-}}=\left\{v_{-}, 0\right\} .
$$

Claim. $u_{+} \in \operatorname{int} C_{+}$and $v_{-} \in-\operatorname{int} C_{+}$are both local minimizers of $\widetilde{\xi}$.

From (4.15) we see that $\widetilde{\xi}_{+}$is coercive. Also, it is weakly lower semicontinuous. So, we can find $y \in W^{1, p}(\Omega)$ such that

$$
\widetilde{\xi}_{+}(y)=\inf \left\{\widetilde{\xi}_{+}(u): u \in W^{1, p}(\Omega)\right\} .
$$

As before, using hypotheses $\mathrm{H}(\mathrm{a})(\mathrm{iv}), \mathrm{H}(\mathrm{f})$ (iii) and the fact that $1<q<\mu, \theta$ we infer that $\widetilde{\xi}_{+}(y)<0=\widetilde{\xi}_{+}(0)$, i.e. $y \neq 0$. But $y \in K_{\widetilde{\xi}}$ (see (4.17)). Hence $y=u_{+}$ (see (4.16)).

Note that $\left.\widetilde{\xi}\right|_{C_{+}}=\left.\widetilde{\xi}_{+}\right|_{C_{+}}$and $u_{+} \in \operatorname{int} C_{+}$, hence $u_{+}$is a local $C^{1}(\bar{\Omega})$ minimizer of $\widetilde{\xi}$, therefore $u_{+}$is a local $W^{1, p}(\Omega)$-mini-mizer of $\widetilde{\xi}$ (see Proposition 2.7). Similarly for $v_{-} \in-\operatorname{int} C_{+}$, using this time the functional $\widetilde{\xi}_{-}$. This proves the Claim.

We may assume that $\widetilde{\xi}\left(v_{-}\right) \leq \widetilde{\xi}\left(u_{+}\right)$(the analysis is similar if the opposite inequality holds). By virtue of the Claim, as in Aizicovici, Papageorgiou and Staicu [1] (see the proof of Proposition 29), we can find $\rho \in(0,1)$ small such that

$$
\widetilde{\xi}_{+}\left(v_{-}\right) \leq \widetilde{\xi}\left(u_{+}\right)<\inf \left\{\widetilde{\xi}(u):\left\|u-u_{+}\right\|=\rho \in W^{1, p}(\Omega)\right\}=\widetilde{\eta}_{\rho} .
$$

Since $\widetilde{\xi}$ is coercive (see (4.15)), it satisfies the PS-condition. This fact and (4.18) permit the use of Theorem 2.1 (the mountain pass theorem). So, we can find $y_{0} \in W^{1, p}(\Omega)$ such that $y_{0} \in K_{\widetilde{\xi}}$ and $\widetilde{\eta}_{\rho} \leq \widetilde{\xi}\left(y_{0}\right)$, hence $y_{0} \notin\left\{v_{-}, u_{+}\right\}$(see (4.18)). Then $y_{0} \in\left[v_{-}, u_{+}\right] \backslash\left\{v_{-}, u_{+}\right\}$(see (4.16)), $y_{0} \in C^{1}(\bar{\Omega})$ (nonlinear regularity) and it solves (1.1).

We may suppose that $y_{0}$ is a critical point of mountain pass type for $\widetilde{\xi}$. Thus,

$$
C_{1}\left(\widetilde{\xi}, y_{0}\right) \neq 0 .
$$

On the other hand, hypothesis H(f)(iii) and Proposition 2.1 of Jiu-Su [11] imply

$$
C_{k}(\widetilde{\xi}, 0)=0 \quad \text { for all } k \geq 0 .
$$

Comparing (4.19) and (4.20), we infer that $y_{0} \neq 0$. Since $y_{0} \in\left[v_{-}, u_{+}\right] \backslash\left\{v_{-}, u_{+}\right\}$, the extremality of $v_{-}$and $u_{+}$implies that $y_{0} \in C^{1}(\bar{\Omega})$ is a nodal solution of (1.1).

We can conclude that the following multiplicity theorem for problem (1.1) is true: 
THEOREM 4.5. If hypotheses $\mathrm{H}(\mathrm{a})$ and $\mathrm{H}(\mathrm{f})$ hold, then problem (1.1) has at least three nontrivial smooth solutions

$$
u_{0} \in \operatorname{int} C_{+}, \quad v_{0} \in-\operatorname{int} C_{+}, \quad \text { and } \quad y_{0} \in C^{1}(\bar{\Omega}), \quad \text { nodal. }
$$

Acknowledgements. The authors whish to thank the referee for his/her corrections and remarks.

The third author gratefully acknowledges the partial support by FEDER funds through COMPETE - Operational Programme Factors of Competitiveness and by Portuguese funds through the Center for Research and Development in Mathematics and Applications and the Portuguese Foundation for Science and Technology (FCT), within project PEst-C/MAT/UI4106/2011 with COMPETE number FCOMP-01-0124-FEDER-022690.

\section{REFERENCES}

[1] S. Aizicovici, N. S. Papageorgiou and V. Staicu, Degree theory for operators of monotone type and nonlinear elliptic equations with inequality constraints, Mem. Amer. Math. Soc. 196 (2008).

[2] Existence of multiple solutions with precise sign information for superlinear Neumann problems, Ann. Mat. Pura Appl. 188 (2009), 679-719.

[3] V. Benci, D. Fortunato and L. Pisani, Solitons like solutions of a Lorentz invariant equation in dimension 3, Rev. Math. Phys. 10 (1998), 315-344.

[4] R. Benguria, H. Brezis And E.H. Lieb, The Thomas-Fermi-von Weizsacker theory of atoms and molecules, Comm. Math. Phys. 79 (1981), 167-180.

[5] Z. Chen And Y. Shen, Infinitely many solutions of Dirichlet problem for p-mean curvature operator, Appl. Math. J. Chinese Univ. Ser. B 18 (2003), 161-172.

[6] J.I. DIAZ AND J.E. SAA, Existence et unicité de solutions positives pour certaines equations elliptiques quasilineaires, C.R. Math. Acad. Sci. Paris 305 (1987), 521-524.

[7] N. Dunford and J. T. Schwartz, Linear Operators, Part I, Interscience, New York, 1958.

[8] L. Gasinski and N.S. Papageorgiou, Nonlinear Analysis, Chapman \& Hall/CRC Press, Boca Raton, 2006.

[9] Anisotropic nonlinear Neumann problems, Calc. Var. Partial Differential Equations 42 (2011), 323-354.

[10] S. Heikkila and V. Lakshmikantham, Monotone Iterative Techniques for Discontinuous Nonlinear Differential Equations, Marcel Dekker, New York, 1994.

[11] Q. JIU AND J. SU, Existence and multiplicity results for perturbations of the p-Laplacian, J. Math. Anal. Appl. 281 (2003), 587-601.

[12] G. Li AND H. Zhou, Multiple solutions to $p$-Laplacian problems with asymptotic nonlinearity as $u^{p-1}$ at infinity, J. London Math. Soc. 65 (2002), 123-138.

[13] G. Lieberman, The natural generalization of the natural conditions of Ladyzhenskaya and Ural'tseva for elliptic equations, Comm. Partial Differential Equations 16 (1991), 311-361.

[14] J. LIU AND J. SU, Remarks on multiple nontrivial solutions for quasilinear resonant problems, J. Math. Anal. Appl. 258 (2001), 209-222. 
[15] S. LiU, Multiple solutions for coercive p-Laplacian equations, J. Math. Anal. Appl. 316 (2006), 229-236.

[16] S.A. Marano And N.S. Papageorgiou, Constant-sign and nodal solutions of coercive $(p, q)$-Laplacian problems, Nonlinear Anal. 77 (2013), 118-129.

[17] D. Motreanu, V. Motreanu and N. S. Papageorgiou, Multiple constant sign and nodal solutions for nonlinear Neumann eigenvalue problems, Ann. Scuola Norm. Sup. Pisa, Cl. Sci. X (2011), 729-755.

[18] D. Motreanu and N. S. Papageorgiou, Multiple solutions for nonlinear Neumann problems driven by a nonhomogeneous differential operator, Proc. Amer. Math. Soc. 139 (2011), 3527-3535.

[19] E. Papageorgiou and N.S. Papageorgiou, A multiplicity theorem for problems with the p-Laplacian, J. Funct. Anal. 244 (2007), 63-77.

[20] N.S. Papageorgiou, E.M. Rocha and V. Staicu, A multiplicity theorem for hemivariational inequalities with a p-Laplacian-like differential operator, Nonlinear Anal. 69 (2008), 1150-1163.

[21] P. Pucci And J. Serrin, The Maximum Principle, Birkhäuser, Basel, 2007.

Sergiu Aizicovici

Department of Mathematics

Ohio University

Athens, OH 45701, USA

E-mail address: aizicovs@ohio.edu

Nikolaos S. Papageorgiou

Department of Mathematics

National Technical University

Zografou Campus

Athens 15780, GREECE

E-mail address: npapg@math.ntua.gr

Vasile Staicu

Department of Mathematics

CIDMA, University of Aveiro

Campus Universitário de Santiago

3810-193 Aveiro, PORTUGAL

E-mail address: vasile@ua.pt 